\title{
Reduction of Isoagglutinin in Intravenous Immunoglobulin (IVIG) Using Blood Group A- and B-Specific Immunoaffinity Chromatography: Industry-Scale Assessment
}

\author{
Simon Gerber ${ }^{1} \cdot$ Annette Gaida $^{1} \cdot$ Nicole Spiegl $^{2} \cdot$ Sandra Wymann $^{1}$ • \\ Adriano Marques Antunes ${ }^{2} \cdot$ Ibrahim El Menyawi $^{1} \cdot$ Brigitte Zurbriggen $^{3}$. \\ Alphonse Hubsch $^{4} \cdot$ Martin Imboden $^{1}$
}

Published online: 19 September 2016

(c) The Author(s) 2016. This article is published with open access at Springerlink.com

\begin{abstract}
Background Hemolysis, a rare but potentially serious complication of intravenous immunoglobulin (IVIG) therapy, is associated with the presence of antibodies to blood groups A and B (isoagglutinins) in the IVIG product. An immunoaffinity chromatography (IAC) step in the production process could decrease isoagglutinin levels in IVIG.

Objectives Our objectives were to compare isoagglutinin levels in a large number of IVIG (Privigen ${ }^{\circledR}$ ) batches produced with or without IAC and to assess the feasibility of the production process with an IAC step on an industrial scale. Methods The IAC column comprised a blend of anti-A and anti-B resins formed by coupling synthetic blood group antigens (A/B-trisaccharides) to a base bead matrix, and was introduced towards the end of the industrial-scale IVIG manufacturing process. Isoagglutinin levels in IVIG were determined by anti-A and anti-B hemagglutinin direct and indirect methods according to the European Pharmacopoeia (Ph. Eur.) and an isoagglutinin flow cytometry assay. IVIG product quality was assessed with respect to the retention of immunoglobulin $G$ ( $\operatorname{IgG}$ ) subclasses,
\end{abstract}

S. Gerber and A. Gaida have contributed equally to the development of this manuscript and both should be considered first authors.

Simon Gerber

simon.gerber@cslbehring.com

1 Department of Research and Development, CSL Behring AG, Wankdorfstrasse 10, 3000 Bern 22, Switzerland

2 Department of Quality Control, CSL Behring AG, Bern, Switzerland

3 Department of Project Management, CSL Behring AG, Bern, Switzerland

4 Department of Medical Affairs, CSL Behring AG, Bern, Switzerland specific antibodies, and removal of IgM using standardized procedures.

Results The IAC step reduced isoagglutinins in IVIG by two to three titer steps compared with lots produced without IAC. The median anti-A and anti-B titers with IAC were 1:8 and $1: 4$, respectively, when measured by the $\mathrm{Ph}$. Eur. direct method, and 1:2 and $<1$, respectively, when measured by the $\mathrm{Ph}$. Eur. indirect method. The isoagglutinin flow cytometry assay showed an 87-90 \% reduction in isoagglutinins in post-IAC versus pre-IAC fractions. IAC alone reduced anti-A and anti-B of the IgMs isotype by 92.5-97.8\% and 95.4-99.2\%, respectively. Other product quality characteristics were similar with and without IAC. Conclusions IAC is an effective method for reducing isoagglutinin levels in IVIG, and it is feasible on an industrial scale.

\section{Key Points}

Intravenous immunoglobulin (IVIG) is commonly used in the effective management of autoimmune and inflammatory diseases.

Hemolysis, a rare but potentially serious complication of IVIG therapy, is associated with the presence of antibodies to blood groups A and B (isoagglutinins) in the IVIG product.

In this study, the introduction of an immunoaffinity chromatography (IAC) step in the IVIG production process reduced isoagglutinins by two to three titer steps compared with lots produced without IAC.

Findings from this study provide strong evidence from a large sample size that IAC is an effective method for reducing isoagglutinin levels in IVIG product and is feasible on an industrial scale. 


\section{Introduction}

Intravenous immunoglobulin (IVIG) treatments-therapeutic preparations of human immunoglobulin $\mathrm{G}(\mathrm{IgG})$ obtained from the plasma of healthy blood donors-were initially developed as a substitution therapy for primary immunodeficiency conditions; however, their use has since expanded to include treatment of autoimmune and inflammatory diseases such as primary immune thrombocytopenia, Guillain-Barré syndrome, chronic inflammatory demyelination polyneuropathy (CIDP), and Kawasaki disease [1]. A well-documented and rare but potentially serious complication of high-dose IVIG therapy is hemolysis, which can result in severe hemolytic anemia [2-6]. Patient risk factors for developing hemolytic anemia following IVIG therapy include having a non-O blood group, the presence of an underlying inflammatory state, and the use of high doses of IVIG [5, 7]. In January 2014, the US FDA sponsored a public workshop to discuss strategies to address hemolytic complications of IVIG infusions, with subsequent publication in 2015 of a special supplement based on the workshop outcomes $[8,9]$.

Clinically significant hemolytic anemia is associated with the presence of antibodies to blood groups A and B (also known as isoagglutinins) in the IVIG product, originating from donor plasma [5, 7]. It has been proposed that the increased incidence of IVIG-related hemolysis in recent years may be a result of the use of not only higher IVIG doses but also modern methods for IVIG production [5]. The original IVIG production methods using Cohn-like ethanol fractionation remove most of the isoagglutinins in a precipitation step (removal of FIII), resulting in products with low isoagglutinin titers [10-12]. Modern production methods have replaced this precipitation step with caprylate fractionation and/or chromatography, which has improved the recovery of IgG from plasma to yield products with high purity and functional integrity but does not reduce isoagglutinins. Consequently, these products have higher titers of isoagglutinins despite a higher general overall purity $[10,13]$

Licensed IVIG products meet regulatory standards for efficacy and tolerability. The current European Pharmacopoeia (Ph. Eur.) requirement for anti-A and anti-B titers, measured by the Ph. Eur. direct method in licensed IVIG products is less than or equal to the World Health Organization (WHO) reference reagent, which has a maximal allowable titer for anti-A and anti-B of 1:64 measured by the same method [14]. However, further reduction of isoagglutinin levels in these products could potentially reduce the risk of IVIG-related hemolytic reactions [13]. Although no maximum titer for isoagglutinins below which the risk of hemolysis is considered low can be defined (and even complete removal of isoagglutinins would not reduce the risk to zero, as other antibodies may be involved) [15], analysis of the EUDRA vigilance database has suggested that IVIG products with a median anti-A of $\leq 1: 16$, measured by the $\mathrm{Ph}$. Eur. direct method, are associated with a lower hemolytic risk [13].

To reduce the levels of isoagglutinins in a chromatographically purified IVIG product (Privigen ${ }^{\circledR}$, CSL Behring AG, Bern, Switzerland), an immunoaffinity chromatography (IAC) method was developed; this process was implemented on an industrial scale in 2015. In addition, an isoagglutinin flow cytometry assay was developed to allow anti-A and anti-B quantification in IVIG production intermediates before and after the IAC step. We report the isoagglutinin levels in a large number of IVIG batches produced with and without the IAC process step.

\section{Methods}

\subsection{Intravenous Immunoglobulin (IVIG) (Privigen) Manufacturing Process}

Figure 1 shows the original and modified processes for industrial-scale manufacture (defined in this study as up to 10,000 L plasma equivalent) of the IVIG product, Privigen.

The original manufacturing process for Privigen has been previously published in detail $[16,17]$. In brief, an IgG-enriched precipitate is obtained following cold ethanol precipitation of pooled donor plasma, which then undergoes principal purification by octanoic acid fractionation. The resulting precipitate, which contains lipids, highmolecular-weight proteins, and proteases, is removed by depth filtration, and the supernatant is further processed by $\mathrm{pH} 4$ incubation to inactivate potentially present viruses. Anion-exchange chromatography (AIEX) is subsequently used to remove residual protein impurities (mainly IgA and IgM) and aggregates, and a second virus-elimination step (virus- or nanofiltration) is applied to ensure the removal of any potentially remaining viruses. The final step is concentration of the IgG product via ultrafiltration-diafiltration and final formulation.

In the modified process for Privigen manufacture, which aims to selectively reduce anti-A and anti-B in the product, all steps in the original process remain the same, and an additional IAC step has been introduced immediately downstream of the AIEX step. For the IAC step, synthetic blood group antigens (A- and B- trisaccharides) were coupled to a base bead matrix to form anti-A- and anti-Bspecific resins; these resins were blended to allow chromatographic reduction of isoagglutinins in one dedicated step. 


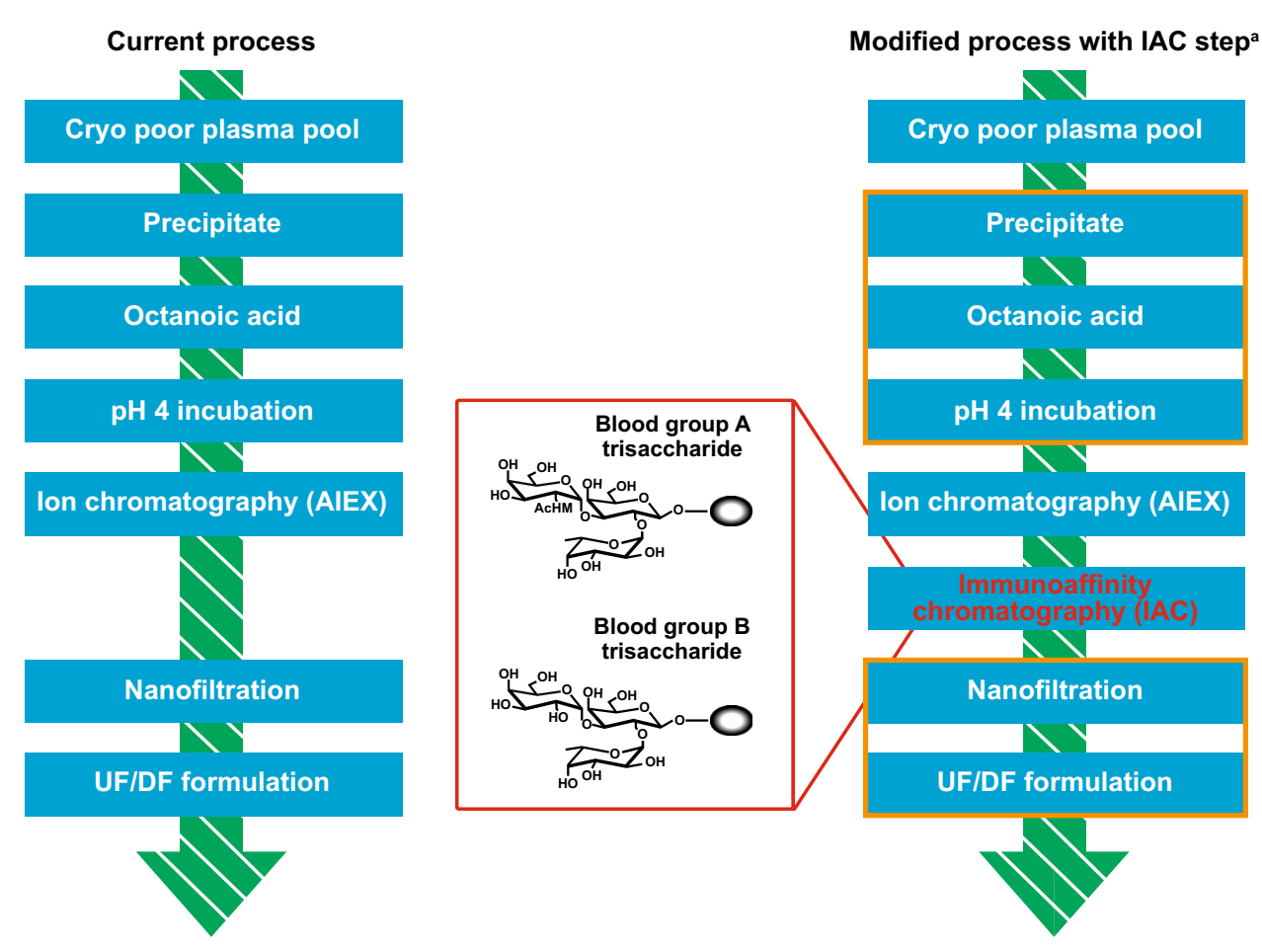

Fig. 1 Original and modified processes for the manufacture of the intravenous immunoglobulin product, Privigen. ${ }^{\text {a } W h e n ~ a s s e s s i n g ~ t h e ~}$ clearance of immunoglobulin $\mathrm{M}$ isoagglutinins by the anion-exchange chromatography and immunoaffinity chromatography steps, the process steps outlined in orange (ethanol- and octanoic acid fractionation) were omitted to minimize the loss of immunoglobulin $\mathrm{M}$, and therefore the loss of the immunoglobulin $\mathrm{M}$ isoagglutinins. This was done because the octanoic acid fractionation step is the main elimination step of total immunoglobulin $\mathrm{M}$. The intention was to

\subsubsection{Anti-A Donor Screening}

A previously published, industrial-scale, high-throughput screening method (OPTISA) was used to identify and exclude plasma donors with high anti-A titers, which have been shown to correlate with high anti-B titers, for the production of IVIG [18]. Over 2.5 years, Privigen was predominantly produced from anti-A screened plasma; about $5 \%$ of donors with the highest anti-A titers were excluded. From October 2015, anti-A donor screening has been progressively replaced with the specific IAC step.

\subsection{IVIG Lots Analyzed}

Isoagglutinin titers were measured in product lots developed as follows: (1) no donor screening, no IAC; (2) donor screening, no IAC; and (3) no donor screening, with IAC. Lots with donor screening and IAC were not developed. Isoagglutinin titers for IVIG lots developed in this study were also compared with those for the IVIG product retain as much immunoglobulin $\mathrm{M}$ as possible for the subsequent isoagglutinin removal steps. AIC immunoaffinity chromatography, AIEX anion exchange chromatography, $D F$ diafiltration, $U F$ ultrafitration. Adapted with permission from Hoefferer et al. [19] (C) 2015 AABB. Hoefferer L, Glauser I, Gaida A, Willimann K, Marques Antunes A, Siani B, et al. Isoagglutinin reduction by a dedicated immunoaffinity chromatography step in the manufacturing process of human immunoglobulin products. Transfusion. 2015;55(Suppl 2): S117-21. doi:10.1111/trf.13088

Sandoglobulin/Carimune ${ }^{\circledR} \quad$ (CSL Behring AG, Bern, Switzerland), which was developed using a Cohn-like fractionation method [12].

\subsection{Immunoglobulin G (IgG) Isoagglutinin Titer Determination}

The IgG isoagglutinin levels in IVIG product lots were assessed using the anti-A and anti-B hemagglutinin direct and indirect methods according to the Ph. Eur. [14]. In addition, anti-A/B levels were quantified using an isoagglutination flow cytometry assay.

\subsubsection{Direct Method According to the European Pharmacopoeia (Ph. Eur.)}

Based on previously described methods [14], $\mathrm{A}_{1}$ or $\mathrm{B}$ red blood cells (RBCs) were treated with papain (Bio-Rad, Hercules, CA, USA) according to manufacturer instructions. Twofold serial dilutions of IVIG final product (starting from $50 \mathrm{~g} / \mathrm{l}$ of total $\mathrm{Ig}$ ) were incubated with the 
papain-treated $\mathrm{A}_{1}$ or $\mathrm{B}$ blood group $\mathrm{RBCs}$ in a microtiter plate. Following centrifugation, microtiter plates were angled at $70^{\circ}$, and the strength of RBC agglutination was visually evaluated based on cell pattern (streaming vs. nonstreaming). The endpoint titer was defined as the highest dilution with visible RBC agglutination (non-streaming pattern). The reference materials used were the WHO reference reagents for hemagglutination tests (positive control 07/306, negative control 07/308, and limit reference preparation 07/310; all from the National Institute for Biological Standards and Control [NIBSC], Potters Bar, Hertfordshire, UK).

\subsubsection{Indirect Method According to the Ph. Eur}

The indirect agglutination test was performed according to Ph. Eur. methods [14]. In brief, twofold serial dilutions of IVIG final product (starting from $30 \mathrm{~g} / \mathrm{l}$ of total $\mathrm{Ig}$ ) were incubated with $A_{1}$ or $B$ blood group RBCs in microtiter plates for $30 \mathrm{~min}$ at $37^{\circ} \mathrm{C}$. Following three wash steps to remove non-specific IgG, anti-human globulin reagent (Pelikloon $^{\mathrm{TM}}$, Sanquin Reagents, Amsterdam, the Netherlands) was added. Agglutination of sedimented RBCs was assessed by macroscopic inspection. The endpoint titer was defined as the highest dilution with a visible RBC agglutination pellet.

\subsubsection{Isoagglutinin Flow Cytometry Assay}

Isoagglutinins were quantified by flow cytometry based on the specific binding of antibodies to blood group A and B RBCs (Inter-regional Blood Donation Service, Bern, Switzerland; pool of three donors each). Intermediate samples were pre-diluted in phosphate-buffered saline (PBS, pH 7.4) containing $1 \%$ bovine serum albumin (BSA, $30 \%$ solution) to an IgG concentration of either $0.1 \mathrm{~g} / 1$ (samples before IAC column) or $0.5 \mathrm{~g} / 1$ (samples after IAC column). RBCs were washed twice in PBS:BSA $1 \%$ buffer and re-suspended to approximately $1.5 \times 10^{4}$ cells/ $\mu$ l. Incubation of samples and cells (50 $\mu \mathrm{l}$ each) was carried out on 96-well microtiter plates for $60 \mathrm{~min}$ at room temperature (RT), followed by two wash cycles using PBS (200 $\mu \mathrm{l} /$ well). Bound isoagglutinins were detected by incubation for $30 \mathrm{~min}$ at RT with $50 \mu \mathrm{l}$ of R-phycoerythrin-conjugated secondary antibody pre-diluted 1:300 in PBS:BSA $1 \%$ (Goat F[ab'] $]_{2}$ anti-human Fc $\gamma$, Jackson ImmunoResearch, West Grove, PA, USA). Plate washing was repeated three times, and cells were re-suspended in $50 \mu \mathrm{l}$ of PBS for detection of fluorescence intensity on FACSCanto-II (Becton-Dickinson AG, Allschwil, Switzerland). Samples were quantified in relation to an equally concentrated internal standard (a selected lot of Privigen), with data presented as percent relative fluorescence intensity (RFI) versus the internal standard.

\subsection{Product Quality Assessments}

\subsubsection{Specific Antibodies Assay}

To evaluate whether the IAC step reduces the concentrations of antibodies other than isoagglutinins, the concentrations of six specific antibodies-to parvovirus B19, hepatitis B surface antigen, streptolysin-O, diphtheria antitoxin, poliomyelitis, and measles_-were compared between IVIG lots manufactured with and without the IAC step. Antibodies against streptolysin-O were quantified by a standard nephelometry assay, antibodies against hepatitis B surface antigen and parvovirus B19 were quantified by standard enzyme immunoassays, and antibodies against polio, diphtheria, and measles were quantified by neutralization assays [19]. In addition, process intermediates before the IAC step (i.e., 'feed' of IAC) and intermediates directly after the IAC step (i.e., 'flow-through' of IAC) were analyzed for pneumococcal capsular polysaccharide IgG and haemophilus influenza B antibodies via commercially available ELISA kits (The Binding Site, Birmingham, UK).

\subsubsection{IgG Subclasses}

Process intermediates before the IAC step (i.e., 'feed' of IAC) and final IVIG product ('bulk') were analyzed for the distribution of IgG subclasses (IgG1, IgG2, IgG3, and IgG4) via nephelometry. Subclasses were expressed as \% of total IgG.

\subsubsection{IgM Isoagglutinin Assay}

A laboratory-scale investigation was performed to investigate the effect of AIEX and IAC on anti-A/B IgM. Three plasma pools with different isoagglutinin titers (1:1024 [high titer], 1:512 [medium titer], and 1:32 [normal titer]), measured with an indirect agglutination test using gel cards (Ortho Clinical Diagnostics [OCD], Rochester, NY, USA), were directly applied to the AIEX and IAC column, omitting the ethanol and octanoic acid fractionation steps, which are the main elimination steps of total $\operatorname{IgM}$ in the Privigen purification process. The intention was to retain as much IgM as possible for the subsequent isoagglutinin-removal steps.

Concentrations of anti-A and anti-B IgM were measured in the starting pools (before IAC) and flow-through fractions of the post-AIEX and post-IAC steps using an isoagglutinin flow cytometry assay. The tested fractions were diluted to the highest possible concentration and incubated with a constant amount of human serum of blood group $\mathrm{AB}$ (to block unspecific binding). The concentrations were chosen to avoid agglutination of the RBCs. After incubation with either blood group A or B RBCs, the cells were washed with Hank's balanced salt solution containing 
$1 \mathrm{mg} / \mathrm{ml} \mathrm{BSA} \mathrm{followed} \mathrm{by} \mathrm{an} \mathrm{incubation} \mathrm{with} \mathrm{a} \mathrm{mono-}$ clonal biotinylated anti-Hu IgM antibody (Nordic Laboratories, the Netherlands). After two further washes with this solution, the cells were incubated with phycoerythrinconjugated streptavidin (Jackson ImmunoResearch). After a final wash, bound IgM isoagglutinins were detected using a FACSCanto-II flow cytometer (Becton-Dickinson AG). The anti-A/anti-B IgM concentration was calculated based on a standard curve of polyclonal IgM. Reduction in IgM isoagglutinin was calculated relative to the isoagglutinin content of the corresponding starting plasma pool.

\subsection{Statistics}

Anti-A and anti-B titers measured by direct and indirect methods (according to the Ph. Eur.) in different IVIGs were compared with exact Chi squared tests (Monte Carlo simulated p-values). Student's $t$-tests with correction for unequal variances were used to assess differences in isoagglutinin levels measured by flow cytometry. $P$ values were corrected for multiple comparisons with Holm's method.

For specific antibodies in IVIGs manufactured with and without IAC or in IVIG precursors before and after IAC, $99 \%$ Student's $t$ confidence intervals (CIs) for the ratio of two mean antibody concentrations were calculated by first taking logarithms of the original data and then exponentiating the resulting boundaries. As there were eight comparisons, an overall significance level of $0.92 \quad(>0.9$, as required for a single comparison) was ensured.

For measures of IgG subclasses in IVIG precursor products in the manufacturing process before IAC and in the final IVIG product, $90 \%$ CIs for the ratio of two means were calculated by first taking logarithms of the original data and then using a Bayesian approach with uninformative priors and multivariate normal likelihoods, resulting in a multivariate Student's $t$ distribution. With this approach, no corrections for multiple testing were required because the joint distribution of $\operatorname{IgG}$ subclasses was considered.

All statistical analyses were performed using the software R (https://www.r-project.org/), version 3.2.1. $P$ values $\leq 0.05$ were considered statistically significant when testing for difference. When testing for equivalence of two mean outcomes, statistical equivalence was assumed when the $90 \%$ CI (99\% CI to account for multiplicity with specific antibody testing results) of the ratio was fully included in the interval $0.8-1.25$.

\section{Results}

A total of 708 historical lots using the original process without anti-A screening or the IAC step were included in this evaluation: 175 lots were produced using the original process with anti-A screened plasma (low anti-A/anti-B titer plasma), and 198 lots were produced using the modified process with the IAC step (using plasma not screened for anti-A).

\subsection{Isoagglutination Titers after Immunoaffinity Chromatography (IAC) or Donor Screening}

\subsubsection{Assessment Using the Direct Method According to the Ph. Eur}

Figure 2 shows isoagglutinin titers in IVIG lots prepared without screening or IAC, with anti-A plasma screening, and with the IAC step, measured by the Ph. Eur. direct method.

Compared with lots produced using the original production process (without IAC or screening), the modified process (with IAC) was associated with a two-step reduction in the median anti-A (from $1: 32$ to $1: 8, p<0.0001$; Fig. 2a) and anti-B (from $1: 16$ to $1: 4, p<0.0001$; Fig. 2b) titers.

Anti-A plasma screening resulted in a modest but statistically significant reduction in isoagglutinin $(p<0.0001$ and $p=0.02$ for anti-A and anti-B, respectively). The median titers were similar in both groups (1:32 and 1:16 for anti-A and anti-B, respectively), but titers below the median became more frequent (Fig. 2a, b).

Some variability in measurements obtained using the $\mathrm{Ph}$. Eur. direct method was noted. The positive control (WHO reference reagent, NIBSC code 07/306) showed a difference of one titer step when the same sample was repeatedly measured; the distributions of titers in the positive control were $55 \%$ at $1: 32$ and $45 \%$ at 1:64 for anti-A and $38 \%$ at $1: 16$ and $62 \%$ at $1: 32$ for anti-B $(n=467)$.

\subsubsection{Assessment Using the Indirect Method According to the Ph. Eur}

Compared with lots produced using the original production process, the modified process with IAC was associated with a three-step reduction in the median anti-A (from 1:16 to $1: 2, p<0.0001$ ) and anti-B (from $1: 8$ to $<1$, $p<0.0001)$ titers when measured by the $\mathrm{Ph}$. Eur. indirect method. This differed from measurements by the $\mathrm{Ph}$. Eur. direct method, which showed a two-step reduction in isoagglutinin titers with IAC.

Use of anti-A screened plasma in the original production process (no IAC) showed a one-step reduction in the median anti-A (from 1:16 to $1: 8, p<0.0001$ ) and anti-B (from $1: 8$ to $1: 4, p<0.0001$ ) titers compared with nonscreened plasma. 
Fig. 2 Anti-A (a) and anti-B (b) titers in final intravenous immunoglobulin product lots, measured by the European Pharmacopoeia direct method. IAC immunoaffinity chromatography, $\operatorname{Ig} G$ immunoglobulin $\mathrm{G}$

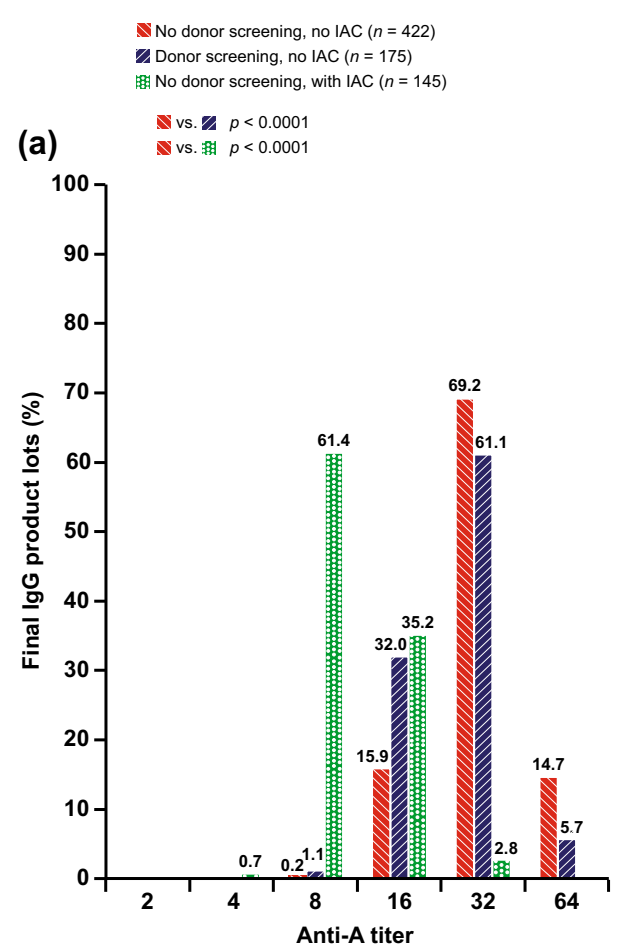

\subsubsection{Assessment Using an Isoagglutinin Flow Cytometry Assay}

The isoagglutinin flow cytometry assay confirmed that the introduction of an IAC step in the production process was associated with a reduction in isoagglutinins in the final IVIG product (Fig. 3). Anti-A and anti-B were reduced after IAC by a median (min, max) of $87 \%(60,92)$ and $90 \%(71,95)$, respectively (both $p<0.0001)$ compared with before IAC $(n=190)$.

This decrease appears consistent with the approximately two- to three-step reduction in titers seen with the agglutination assays according to the Ph. Eur., although the correlation between these methods has not been investigated.

\subsection{Isoagglutination Titers After IAC vs. Cohn-Like Fractionation}

Figure 4 shows isoagglutinin titers, measured using the $\mathrm{Ph}$. Eur. direct method, in IVIG lots produced with the IAC step (Privigen) and in IVIG lots produced using Cohn-like fractionation (Sandoglobulin/Carimune), which removes isoagglutinins with a precipitation step (separation of FIII) rather than IAC.

When measured by the $\mathrm{Ph}$. Eur. direct method, the median anti-A and anti-B titers for IVIG lots produced by Cohn-like fractionation were $1: 8$ and 1:4, respectively. In lots of IVIG produced with an IAC step, median anti-A and anti-B titers were also $1: 8$ and 1:4, respectively. Lower isoagglutinin titers tended to be more frequent in IVIG produced by Cohn-like fractionation, and this difference reached statistical significance for anti-B $(p=0.0006)$ but not for anti-A $(p=0.07)$.

When measured by the $\mathrm{Ph}$. Eur. indirect method, the median anti-A titers were $<1$ and 1:1 $(p<0.0001)$ in IVIG lots produced by Cohn-like fractionation and those produced with the IAC step, respectively. Anti-B titers were $<1$ in both IVIG products $(p=0.71)$.

\subsection{Product Characteristics and Quality After IAC}

\subsubsection{Specific Antibodies}

Table 1 shows the concentrations of specific antibodies to parvovirus B19, hepatitis B surface antigen, streptolysin-O, diphtheria antitoxin, poliomyelitis, and measles in lots of IVIG. In addition, specific antibodies to bacteria that have an outer capsule composed of polysaccharides (pneumococcal capsular polysaccharide $\operatorname{IgG}$ and haemophilus influenza B) were analyzed in two intermediates of the IVIG production process: the intermediate before entering the IAC column (i.e., 'feed') and the intermediate after passing the IAC column (i.e., 'flow-through'). The $99 \%$ CIs for the ratios of the geometric means of specific antibody concentrations in IVIG lots with and without IAC or 
Before IAC $(n=190)$

$\square$ After IAC $(n=190)$

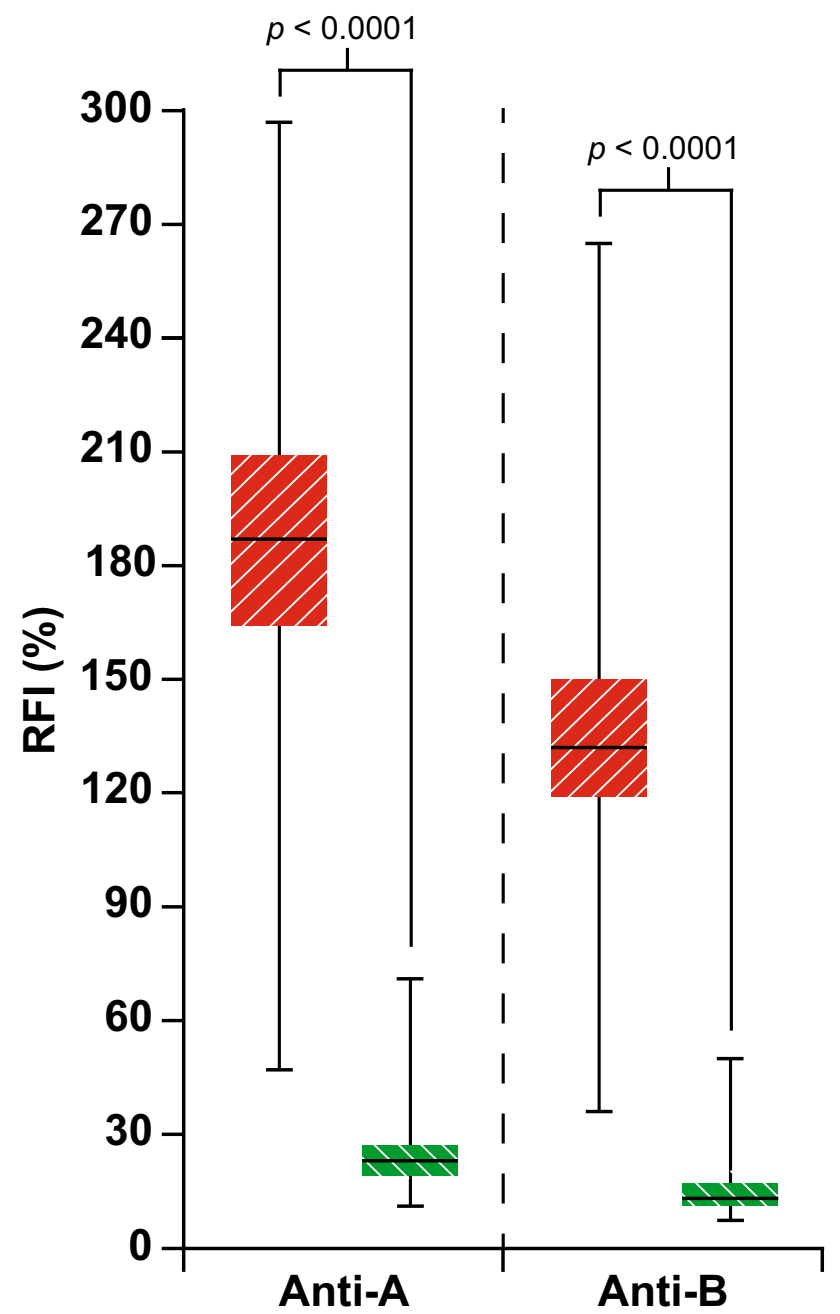

Fig. 3 Immunoglobulin $G$ isoagglutinin levels with and without the immunoaffinity chromatography step in the production process, measured using an isoagglutinin flow cytometry assay. Anti-A and anti-B relative fluorescence intensity, measured relative to the internal standard. RFI relative fluorescence intensity, IAC immunoaffinity chromatography

in intermediates before and after IAC were within the range of $0.8-1.25$, with the exception of anti-parvovirus B19, for which the upper limit of the $99 \%$ CI was 1.255 (Table 1).

\subsubsection{IgG Subclass Distribution}

The distributions of IgG subclasses were similar before and after the IAC step, i.e., the $90 \%$ CIs for the ratios of the geometric means of $\mathrm{IgG}$ subclasses in process intermediate before IAC and in the final IVIG product were all within the range of $0.8-1.25$ (Table 2).

\subsubsection{IgM Isoagglutinin Levels}

Results from an IgM isoagglutinin flow cytometry assay showed that AIEX and IAC were effective at reducing both anti-A and anti-B IgM concentrations in IVIG product (Table 3). Compared with starting plasma pools, the IAC step alone reduced anti-A IgM concentrations by $92.5-97.8 \%$ and reduced anti-B $\operatorname{IgM}$ by $95.4-99.2 \%$ across the three plasma pools. The AIEX and IAC steps together reduced anti-A IgM by $96.4-99.4 \%$ and reduced anti-B IgM by $97.9-99.4 \%$ compared with starting plasma pools.

\section{Discussion}

This industrial-scale study showed that inclusion of a dedicated IAC step towards the end of the IVIG production process reduced anti-A and anti-B titers in the final product by up to two to three titer steps, or 87-90\%, compared with lots produced without IAC. These findings support and expand upon those reported in a small study that also showed a three-titer step, or $88-90 \%$, reduction in isoagglutinins in ten lots of Privigen/Hizentra ${ }^{\circledR}$ produced with IAC [19]. The study reported here analyzed almost 200 IVIG lots produced using IAC, showing that the reduction in isoagglutinin levels of two to three titer steps with IAC is robust and consistent. Furthermore, the modified process including the IAC step is feasible at industrial-scale production levels. The ability of IAC to effectively reduce isoagglutinin levels has also been shown by Dhainaut et al. [20], who reported lower concentrations of anti-A and anti$\mathrm{B}$ in another IVIG manufactured using an IAC step compared with IVIG products produced without IAC.

When measured by the Ph. Eur. direct method, IVIG lots produced with IAC showed median anti-A and anti-B titers of $1: 8$ and $1: 4$, respectively. These titers were comparable to those obtained in this study for IVIG lots produced using a Cohn-like fractionation method, including the separation of FIII, which is known to remove isoagglutinins [10,13].

IVIG products can be divided into two groups according to the isoagglutinin content: IVIG purified using a chromatographic process with an anti-A titer of $>1: 16$ (measured by the $\mathrm{Ph}$. Eur. direct method) and IVIG produced with a Cohn-like ethanol fractionation process with an antiA titer of $\leq 1: 16$ [13]. The difference between these two product groups is similar for anti-B titer $(>1: 8$ and $\leq 1: 8$, respectively). Privigen produced with the IAC step would be among the IVIG products with the lowest isoagglutinin levels. Consistently, another IVIG product produced using a chromatographic process including an IAC isoagglutinin depletion step in a different study also had isoagglutinin 
Fig. 4 Anti-A and anti-B titers in intravenous immunoglobulin lots produced with the immunoaffinity chromatography step (Privigen) and intravenous immunoglobulin lots produced using Cohn-like fractionation (Sandoglobulin/Carimune), measured by the European Pharmacopoeia direct method. $I A C$ immunoaffinity chromatography, $\operatorname{Ig} G$ immunoglobulin G, IVIG intravenous immunoglobulin
缃 No donor screening, with IAC ( $n=145)$

$\mathbb{N}$ IVIG produced using Cohn-like fractionation $(n=119)$

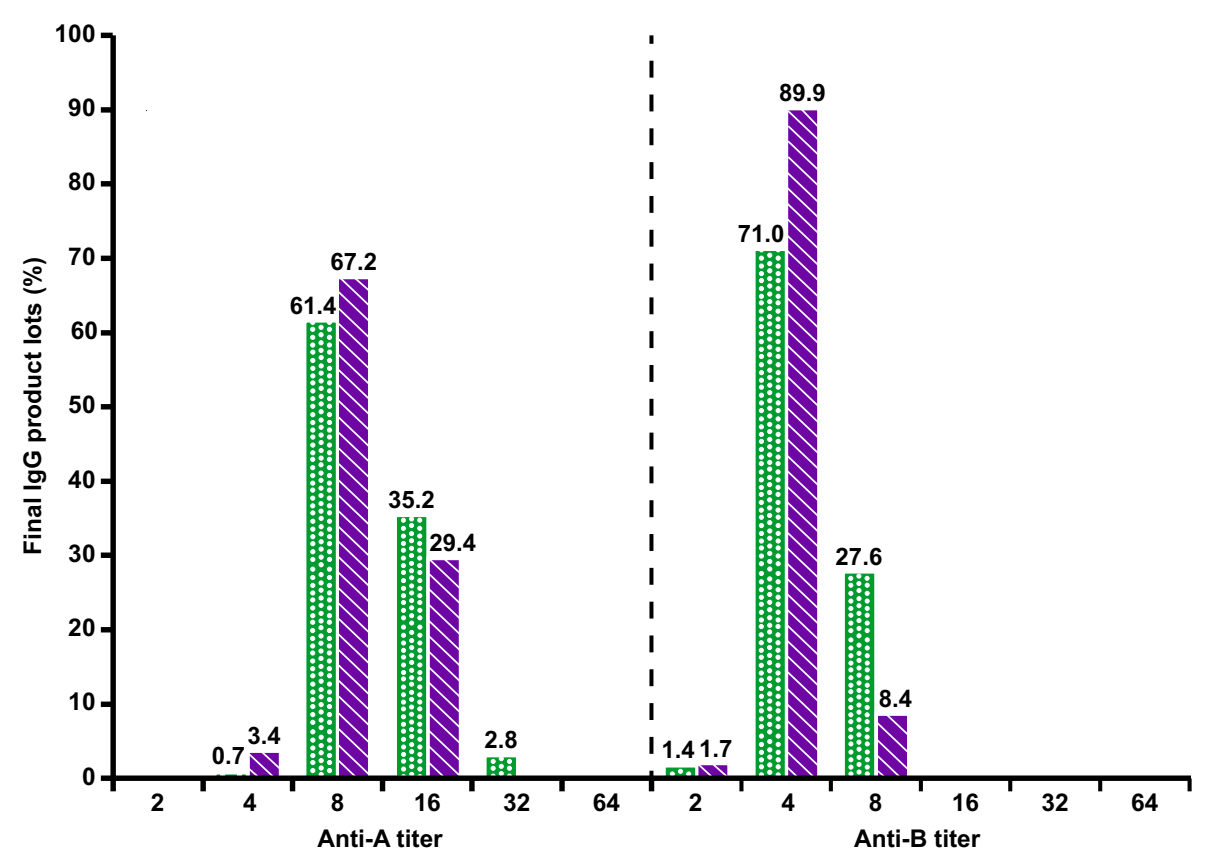

levels as low as IVIGs produced with a Cohn-like process [21].

It is worth noting that the median anti-A isoagglutinin titer of 1:8 (measured by the $\mathrm{Ph}$. Eur. direct method) in IVIG lots produced with IAC reported here is lower than the median titer for anti-A of $\leq 1: 16$, which has been suggested to be associated with a lower risk of hemolytic reactions [13]. However, only complete removal of antibodies against the $\mathrm{ABO}$ antigens would eliminate the risk of ABO-mediated hemolytic reactions, which would still not eliminate a potential risk of hemolysis mediated by antibodies against other erythrocyte antigens [15]. Further studies are required to ascertain whether the lower isoagglutinin titer in IVIG produced with IAC translates into a lower incidence of hemolysis and hemolytic anemia. An observational clinical trial (Privigen PASS; EU post-authorization study [PAS] register number ENCePP/SDPP/ 6040) is currently underway to address this question [22].

Extrapolating from the isoagglutinin content of an IVIG product to the risk of hemolysis is further complicated by the imprecision of the assays available to quantify isoagglutinins. Results using the Ph. Eur. direct method can vary as much as one titer step when repeatedly measuring the same sample. Indeed, in our laboratories, the positive control included in every assay was found to give an anti-A result of 1:32 in $55 \%$ of assays and 1:64 in $45 \%$ of assays; the result for anti-B was $1: 16$ in $38 \%$ and $1: 32$ in $62 \%$ of assays. In this study, the reduction in isoagglutinin titers in IVIG lots prepared with IAC, compared with lots produced without IAC, was confirmed by measuring anti-A and anti$\mathrm{B}$ levels using three different assays: the $\mathrm{Ph}$. Eur. direct and indirect methods and an isoagglutinin flow cytometry assay. The $\mathrm{Ph}$. Eur. direct and indirect methods are simple and commonly used; however, their reliance on visual reading of the results by the investigator can mean they are subject to some degree of inter-assay variation [23]. Furthermore, differences in methodologies for the Ph. Eur. direct and indirect agglutination tests can result in differences in isoagglutinin titer results [21], as seen here with the different median anti-A and anti-B titers reported for IVIG measured by the Ph. Eur. direct and indirect tests. Therefore, isoagglutinin levels were also assessed using an isoagglutinin assay by flow cytometry. While the results of these assays cannot be compared directly, all three assays showed that the IAC step was associated with a decrease in IVIG isoagglutinin titers. In addition, the isoagglutinin flow cytometry assay results were in alignment with those observed using the $\mathrm{Ph}$. Eur. indirect agglutination test; isoagglutinin levels were reduced by $87-90 \%$, which is consistent with a three-titer step reduction.

It is generally accepted that $\operatorname{IgM}$ and IgG antibodies to $\mathrm{A}$ and $\mathrm{B}$ blood group antigens are present in human serum [24]. IgM isoagglutinins are considered to have a higher hemolytic potential as IgM is a strong activator of complement $[15,25]$. Current IVIG products contain only traces of $\operatorname{IgM}$ (e.g., $\leq 10 \mathrm{mg} / \mathrm{l}$ for Privigen); however, their IgM isoagglutinin content has generally not been investigated. In the original manufacturing process for Privigen, 
Table 1 Specific antibody concentrations in intravenous immunoglobulin lots produced with and without the immunoaffinity chromatography step and in intermediates ('feed' and 'flow-through') of immunoaffinity chromatography

\begin{tabular}{|c|c|c|c|c|c|}
\hline \multirow[t]{2}{*}{ Specific antibody } & \multirow[t]{2}{*}{ Process $^{\mathrm{a}}$} & \multicolumn{4}{|c|}{ Antibody concentration in IVIG lots (IU/mL) } \\
\hline & & $n$ & Mean (SD) & Median & $99 \% \mathrm{CI}^{\mathrm{c}}$ \\
\hline \multirow[t]{2}{*}{ Anti-streptolysin-O } & Without IAC & 751 & $1684(180)$ & 1740 & \multirow[t]{2}{*}{$0.940-0.991$} \\
\hline & With IAC & 148 & $1625(169)$ & 1670 & \\
\hline \multirow[t]{2}{*}{ Anti-diphtheria toxin } & Without IAC & 751 & $7.2(1.8)$ & 8.0 & \multirow[t]{2}{*}{$1.035-1.171$} \\
\hline & With IAC & 148 & $7.9(1.6)$ & 8.0 & \\
\hline \multirow[t]{2}{*}{ Anti-polio type 1} & Without IAC & 751 & $36.3(26.4)$ & 26.0 & \multirow[t]{2}{*}{$0.870-1.082$} \\
\hline & With IAC & 148 & $34.2(25.1)$ & 26.0 & \\
\hline \multirow[t]{2}{*}{ Anti-parvovirus B19 } & Without IAC & 751 & $289(57)$ & 284 & \multirow[t]{2}{*}{$1.160-1.255$} \\
\hline & With IAC & 148 & $347(55)$ & 345 & \\
\hline \multirow[t]{2}{*}{ Anti-HBS antigen } & Without IAC & 751 & $6.5(3.2)$ & 6.0 & \multirow[t]{2}{*}{$0.961-1.132$} \\
\hline & With IAC & 148 & $6.7(2.8)$ & 6.3 & \\
\hline \multirow[t]{2}{*}{ Anti-measles } & Without IAC & 335 & $19.1(5.8)$ & 17.0 & \multirow[t]{2}{*}{$0.991-1.185$} \\
\hline & With IAC & 86 & $20.6(5.8)$ & 18.0 & \\
\hline \multirow[t]{2}{*}{ Specific antibody } & \multirow[t]{2}{*}{ Step $^{\mathrm{b}}$} & \multicolumn{4}{|c|}{ Antibody concentration in intermediates $(\mathrm{mg} / \mathrm{g})$} \\
\hline & & $n$ & Mean (SD) & Median & $99 \% \mathrm{CI}^{\mathrm{c}}$ \\
\hline \multirow{2}{*}{$\begin{array}{l}\text { Anti-pneumococcal } \\
\text { capsular protein }\end{array}$} & Feed of IAC column & 20 & $7.0(1.1)$ & 6.8 & \multirow[t]{2}{*}{$0.888-1.006$} \\
\hline & Flow-through & 20 & $6.7(1.2)$ & 6.4 & \\
\hline \multirow{2}{*}{$\begin{array}{l}\text { Anti-haemophilus } \\
\text { influenza B }\end{array}$} & Feed of IAC column & 20 & $0.38(0.05)$ & 0.37 & \multirow[t]{2}{*}{$0.918-1.101$} \\
\hline & Flow-through & 20 & $0.37(0.04)$ & 0.35 & \\
\hline
\end{tabular}

$C I$ confidence interval, $H B S$ hepatitis B surface, IAC immunoaffinity chromatography, $I V I G$ intravenous immunoglobulin, $S D$ standard deviation

${ }^{\text {a }}$ Lots were prepared by two different processes: before ('without IAC') and after ('with IAC') implementation of the IAC step in the production process

b Analysis of intermediates before ('feed of IAC column') and after ('flow-through') loading onto the IAC column; both intermediates were from the same lot and consequently the same process (i.e. after the implementation of the IAC step)

c $99 \%$ Student's $t$ CIs for the ratio of the two mean antibody concentrations, calculated by first taking logarithms of the original data and then exponentiating the resulting boundaries. Equivalence is accepted when the $\mathrm{CI}$ is within $0.8-1.25$. As there were eight comparisons, an overall significance level of $0.92(>0.9$, as required for a single comparison) is ensured by the use of $99 \%$ CIs

Table 2 Distribution of immunoglobulin $\mathrm{G}$ subclasses in intermediates ('feed') before immunoaffinity chromatography and final intravenous immunoglobulin product ('bulk')

\begin{tabular}{|c|c|c|c|c|}
\hline \multirow[t]{2}{*}{ IgG subclass } & \multirow[t]{2}{*}{ Process $^{\mathrm{a}}$} & \multicolumn{3}{|c|}{ Distribution of IgG subclass in intermediates (\%) } \\
\hline & & $n$ & Mean (SD) & $90 \% \mathrm{CI}^{\mathrm{b}}$ \\
\hline \multirow[t]{2}{*}{ IgG1 } & Feed of IAC column & 11 & $70.7 \%(1.8)$ & $0.998-1.057$ \\
\hline & IVIG bulk & 11 & $69.8 \%(1.8)$ & \\
\hline \multirow[t]{2}{*}{$\mathrm{IgG} 2$} & Feed of IAC column & 11 & $25.5 \%(1.9)$ & $0.874-1.001$ \\
\hline & IVIG bulk & 11 & $26.3 \%(2.0)$ & \\
\hline \multirow[t]{2}{*}{ IgG3 } & Feed of IAC column & 11 & $2.0 \%(0.3)$ & $0.910-1.041$ \\
\hline & IVIG bulk & 11 & $2.1 \%(0.5)$ & \\
\hline \multirow[t]{2}{*}{ IgG4 } & Feed of IAC column & 11 & $1.8 \%(0.1)$ & $0.911-1.087$ \\
\hline & IVIG bulk & 11 & $1.9 \%(0.2)$ & \\
\hline
\end{tabular}

$C I$ confidence interval, $I A C$ immunoaffinity chromatography, $I g G$ immunoglobulin $\mathrm{G}, I V I G$ intravenous immunoglobulin, $S D$ standard deviation

a Analysis of intermediates before ('feed of IAC column') and after ('IVIG bulk') loading onto the IAC column; both intermediates were from the same lot and consequently the same process (i.e., after the implementation of the IAC step)

b $90 \%$ CIs for the ratio of two means were calculated by first taking logarithms of the original data and then using a Bayesian approach with uninformative priors and multivariate normal likelihoods, resulting in a multivariate Student's $t$ distribution. Equivalence is accepted when the CI is within $0.8-1.25$. No corrections for multiple testing were required since the joint distribution of $\operatorname{IgG}$ subclasses is considered 
Table 3 Immunoglobulin $M$ isoagglutinin levels in plasma pools before and after chromatography (anion exchange and/or immunoaffinity chromatography) steps, measured using an isoagglutinin flow cytometry assay

\begin{tabular}{|c|c|c|c|c|c|c|}
\hline \multirow[t]{2}{*}{$\begin{array}{l}\text { Isoagglutinin content } \\
\text { in plasma pool }\end{array}$} & \multicolumn{3}{|c|}{$\begin{array}{l}\text { Reduction of anti-A IgM (\% of concentration in starting } \\
\text { plasma pool) }\end{array}$} & \multicolumn{3}{|c|}{$\begin{array}{l}\text { Reduction of anti-B IgM (\% of concentration in starting } \\
\text { plasma pool) }\end{array}$} \\
\hline & $\begin{array}{l}\text { Plasma pool } \\
\text { post-AIEX }\end{array}$ & $\begin{array}{l}\text { Plasma pool post- } \\
\text { AIEX and IAC }\end{array}$ & $\begin{array}{l}\text { Plasma pool post- } \\
\text { IAC without AIEX }\end{array}$ & $\begin{array}{l}\text { Plasma pool } \\
\text { post-AIEX }\end{array}$ & $\begin{array}{l}\text { Plasma pool post- } \\
\text { AIEX and IAC }\end{array}$ & $\begin{array}{l}\text { Plasma pool post- } \\
\text { IAC without AIEX }\end{array}$ \\
\hline $\operatorname{High}^{\mathrm{a}}$ & 20.5 & 99.4 & 97.8 & 34.8 & 99.4 & 99.2 \\
\hline Medium $^{\mathrm{a}}$ & 35.5 & 99.1 & 96.5 & 34.8 & 99.2 & 98.8 \\
\hline Normal $^{\mathrm{a}}$ & 66.8 & 96.4 & 92.5 & 51.5 & 97.7 & 95.4 \\
\hline
\end{tabular}

Reduction was calculated relative to the isoagglutinin content of the corresponding plasma pool

AIEX anion exchange chromatography, IAC immunoaffinity chromatography, IgM immunoglobulin M, OCD Ortho Clinical Diagnostics

${ }^{a}$ High, medium, and normal correspond to anti-A isoagglutinin titers of 1:1024, 1:512, and 1:32, respectively, measured by an indirect agglutination test using gel cards (OCD, Rochester, NY, USA)

IgM are mainly removed by the octanoic acid fractionation and AIEX steps [26], resulting in the final low IgM concentration of $\leq 10 \mathrm{mg} / \mathrm{l}$. In a laboratory-scale investigation in this study, the IAC column was shown to further remove IgM isoagglutinins from the final product, resulting in 96.4-99.4 and 97.9-99.4\% reductions in anti-A and anti-B IgM, respectively, after both AIEX and IAC.

Importantly, the IAC step appeared to reduce isoagglutinin levels selectively without otherwise modifying the final product, as indicated by the unaltered distributions of IgG subclasses and the unaltered concentrations of the eight pathogen-specific antibodies tested in this study. The IgG subclass distribution in Privigen remains similar to that in normal human plasma [26]. The $99 \%$ CIs for the ratios of the geometric means of specific antibody concentrations in IVIG lots with and without IAC or in intermediates before and after IAC were within the range of 0.8-1.25, with the exception of anti-parvovirus B19, for which the upper limit of the $99 \%$ CI was 1.255. These deviations are less than or similar to the lot-to-lot variability in IVIGs [27] and are not expected to have clinical consequences. Two of these antibodies (anti-haemophilus B and anti-pneumococcus antibodies) were chosen due to their recognition of polysaccharides on bacterial outer capsules. As the IAC resin is composed of trisaccharide ligands, there may be a concern that specific anti-bacterial antibodies could be removed by the column as well as isoagglutinins [28]. The data presented here provide some reassurance that the modified manufacturing process with IAC does not negatively affect final product quality.

A previous study has shown that screening plasma donors to identify those with high anti-A titers, and subsequent exclusion of their plasma from IVIG production (resulting in exclusion of $5 \%$ of first-time plasma donors), leads to a lesser reduction in isoagglutinin levels in IVIG product [18] (one titer step by the $\mathrm{Ph}$. Eur. indirect agglutination test). The findings from the current study concur with these findings, i.e., a modest reduction in anti-A and anti-B can be achieved with donor screening with exclusion of only a small proportion of potential donors. However, a greater reduction in isoagglutinin titer can be achieved with IAC. Furthermore, IAC works independently of the plasma source, whereas anti-A donor screening may be more difficult to implement, since a large part of the plasma collection process is outside the control of the IVIG manufacturers. In addition, it is doubtful whether an anti-A donor screening program on top of a production process with an isoagglutinin-reduction step (IAC or precipitation steps in the Cohn process) would produce an additional clinically relevant isoagglutinin reduction. Given these considerations, we did not investigate the combination of anti-A donor screening and IAC on an industrial scale.

\section{Conclusion}

Findings from this study provide strong evidence from a large sample size that IAC is an effective method for reducing isoagglutinin levels in IVIG product and is feasible on an industrial scale.

\section{Compliance with Ethical Standards}

This article does not contain any new studies with human or animal subjects performed by any of the authors. Plasma donors gave written consent that their plasma may be used for research purposes.

Funding This study was funded by CSL Behring AG. Medical writing assistance was provided by Vanessa Cobb and Angela Corstorphine of Kstorfin Medical Communications Ltd, supported by CSL Behring AG.

Conflict of interest Simon Gerber, Annette Gaida, Nicole Spiegl, Sandra Wymann, Adriano Marques Antunes, Ibrahim El Menyawi, Brigitte Zurbriggen, Alphonse Hubsch, and Martin Imboden are employees of CSL Behring AG. Alphonse Hubsch, Brigitte Zurbriggen, and Martin Imboden own stocks in CSL Behring. Simon Gerber, Annette Gaida, Nicole Spiegl, Sandra Wymann, Adriano 
Marques Antunes, and Ibrahim El Menyawi have no further conflicts of interest.

Author contributions Simon Gerber: process development of IAC step; compilation, analysis, and interpretation of data; manuscript drafting. Annette Gaida: development of IAC step; compilation, analysis, and interpretation of data; manuscript drafting. Sandra Wymann: development of isoagglutinin flow cytometry assays for IgG and IgM anti-A/B isoagglutinins; manuscript drafting. Ibrahim El Menyawi: process development of IAC step; generation of IgM anti$\mathrm{A} / \mathrm{B}$ isoagglutinins experimental data; manuscript drafting. Brigitte Zurbriggen: project management; manuscript drafting. Alphonse Hubsch: analysis and interpretation of data; manuscript drafting. Nicole Spiegl: support for development and implementation of the isoagglutinin flow cytometry assay in quality control; manuscript drafting. Adriano Marques Antunes: development and implementation of the isoagglutinin flow cytometry assay in quality control; manuscript drafting. Martin Imboden: process development for the IAC step; manuscript drafting.

Open Access This article is distributed under the terms of the Creative Commons Attribution-NonCommercial 4.0 International License (http://creativecommons.org/licenses/by-nc/4.0/), which permits any noncommercial use, distribution, and reproduction in any medium, provided you give appropriate credit to the original author(s) and the source, provide a link to the Creative Commons license, and indicate if changes were made.

\section{References}

1. Hartung HP, Mouthon L, Ahmed R, Jordan S, Laupland KB, Jolles S. Clinical applications of intravenous immunoglobulins (IVIg)—beyond immunodeficiencies and neurology. Clin Experiment Immunol. 2009;158(Suppl 1):23-33.

2. Baxley A, Akhtari M. Hematologic toxicities associated with intravenous immunoglobulin therapy. Int Immunopharmacol. 2011;11:1663-7.

3. Berger M. Adverse effects of IgG therapy. J Allergy Clin Immunol Pract. 2013;1:558-66.

4. Daw Z, Padmore R, Neurath D, Cober N, Tokessy M, Desjardins $\mathrm{D}$, et al. Hemolytic transfusion reactions after administration of intravenous immune (gamma) globulin: a case series analysis. Transfusion. 2008;48:1598-601.

5. Kahwaji J, Barker E, Pepkowitz S, Klapper E, Villicana R, Peng $A$, et al. Acute hemolysis after high-dose intravenous immunoglobulin therapy in highly HLA sensitized patients. Clin J Am Soc Nephrol. 2009;4:1993-7.

6. Wilson JR, Bhoopalam H, Fisher M. Hemolytic anemia associated with intravenous immunoglobulin. Muscle Nerve. 1997;20:1142-5.

7. Padmore RF. Hemolysis upon intravenous immunoglobulin transfusion. Transfus Apher Sci. 2012;46:93-6.

8. US Food and Drug Administration. Public Workshop: Strategies to Address Hemolytic Complications of Immune Globulin Infusions. 2014. http://www.fda.gov/BiologicsBloodVaccines/News Events/WorkshopsMeetingsConferences/ucm378388.htm. Accessed 14 July 2016.

9. Special Issue: Hemolysis Supplement Strategies to Address Hemolytic Complications of Immune Globulin Infusions. Transfusion. 2015;55:i-ii, S1-S127.

10. Romberg V, Hoefferer L, El Menyawi I. Effects of the manufacturing process on the anti-A isoagglutinin titers in intravenous immunoglobulin products. Transfusion. 2015;55(Suppl 2): S105-9.
11. Cohn EJ, Strong LE, et al. Preparation and properties of serum and plasma proteins; a system for the separation into fractions of the protein and lipoprotein components of biological tissues and fluids. J Am Chem Soc. 1946;68:459-75.

12. Kistler P, Nitschmann H. Large scale production of human plasma fractions. Eight years experience with the alcohol fractionation procedure of Nitschmann, Kistler and Lergier. Vox Sang. 1962;7:414-24.

13. Bellac CL, Hottiger T, Jutzi MP, Bogli-Stuber K, Sanger M, Hanschmann KM, et al. The role of isoagglutinins in intravenous immunoglobulin-related hemolysis. Transfusion. 2015;55(Suppl 2):S13-22.

14. Chapter 2.6.20: Anti-A and Anti-B Haemagglutinins, Method A and B. European Pharmacopoeia (PhEur). 8th edition (88); 2016. p. 203-4.

15. Flegel WA. Pathogenesis and mechanisms of antibody-mediated hemolysis. Transfusion. 2015;55(Suppl 2):S47-58.

16. Stucki M, Boschetti N, Schafer W, Hostettler T, Kasermann F, Nowak T, et al. Investigations of prion and virus safety of a new liquid IVIG product. Biologicals. 2008;36:239-47.

17. Komenda M, Stadler D, Malinas T, Moses M, Pragst I, Herzog E, et al. Assessment of the ability of the Privigen(R) purification process to deplete thrombogenic factor XIa from plasma. Vox Sang. 2014;107:26-36.

18. Siani B, Willimann K, Wymann S, Marques AA, Widmer E. Isoagglutinin reduction in human immunoglobulin products by donor screening. Biol Ther. 2014;4:15-26.

19. Hoefferer L, Glauser I, Gaida A, Willimann K, Marques Antunes A, Siani B, et al. Isoagglutinin reduction by a dedicated immunoaffinity chromatography step in the manufacturing process of human immunoglobulin products. Transfusion. 2015; 55(Suppl 2):S117-21. doi:10.1111/trf.13088

20. Dhainaut F, Guillaumat PO, Dib H, Perret G, Sauger A, de Coupade $\mathrm{C}$, et al. In vitro and in vivo properties differ among liquid intravenous immunoglobulin preparations. Vox Sang. 2013;104:115-26.

21. Bellac CL, Polatti D, Hottiger T, Girard P, Sanger M, Gilgen M. Anti-A and anti-B haemagglutinin levels in intravenous immunoglobulins: are they on the rise? A comparison of four different analysis methods and six products. Biologicals. 2014;42:57-64.

22. ENCePP. Privigen ${ }^{\circledR}$ use and haemolytic anaemia in adults and children and the Privigen ${ }^{\circledR}$ safety profile in children with CIDP an observational hospital-based cohort study in the US (Privigen PASS). http://www.encepp.eu/encepp/viewResource.htm?id=6515. Accessed 28 Feb 2016.

23. Aubert D, Foudrinier F, Kaltenbach ML, Guyot-Walser D, MarxChemla C, Geers R, et al. Automated reading and processing of quantitative $\operatorname{IgG}, \operatorname{IgM}, \operatorname{IgA}$, and IgE isotypic agglutination results in microplates. Development and application in parasitologymycology. J Immunol Methods. 1995;186:323-8.

24. Spalter SH, Kaveri SV, Bonnin E, Mani JC, Cartron JP, Kazatchkine MD. Normal human serum contains natural antibodies reactive with autologous $\mathrm{ABO}$ blood group antigens. Blood. 1999;93:4418-24.

25. Wouters D, Zeerleder S. Complement inhibitors to treat IgMmediated autoimmune hemolysis. Haematologica. 2015;100: 1388-95.

26. Berger M. L-proline-stabilized human IgG: Privigen(R) $10 \%$ for intravenous use and Hizentra(R) $20 \%$ for subcutaneous use. Immunotherapy. 2011;3:163-76.

27. Simon HU, Späth PJ. IVIG: mechanisms of action. Allergy. 2003;58:543-52.

28. Spath PJ, Granata G, La Marra F, Kuijpers TW, Quinti I. On the dark side of therapies with immunoglobulin concentrates: the adverse events. Front Immunol. 2015;6:11. 